\title{
Efecto de la mastitis y el estrés sobre la reproducción de la vaca
}

\author{
Córdova-Izquierdo, A. .; Córdova-Jiménez, C.A. ${ }^{2}$; Córdova-Jiménez, M.S. ${ }^{3}$; \\ Saltijeral-Oaxaca, J.A. ${ }^{1}$; Ruiz-Lang, C.G. ${ }^{1}$; Xolalpa-Campos, V.M. ${ }^{1}$; \\ Cortés-Suárez, S. ${ }^{\text {; }}$ Guerra-Liera, J.E. ${ }^{4}$ \\ ${ }^{1}$ Departamento de Producción Agrícola y Animal, Universidad Autónoma Metropolitana, Unidad Xochimilco, \\ México, E-mail: aci57@prodigy.net.mx. ${ }^{2}$ Doctorando de la Facultad de Veterinaria, Universidad de León \\ (España), becario de CONACYT-México. ${ }^{3}$ Laboratorios Brovel SACV. ${ }^{4} F a c$. Agronomía, \\ Univ. Autónoma de Sinaloa, México.
}

\begin{abstract}
Resumen
Córdova-Izquierdo, A.; Córdova-Jiménez, C.A.; Córdova-Jiménez, M.S.; SaltijeralOaxaca, J.A.; Ruiz-Lang, C.G.; Xolalpa-Campos, V.M.; Cortés-Suárez, S.; GuerraLiera, J.E.: Efecto de la mastitis y el estrés sobre la reproducción de la vaca. Rev. vet. 19: 2, 161-166, 2008. Debido a los innegables adelantos genéticos y nutricionales, la industria lechera puede hoy producir con alta eficiencia mayor cantidad de leche por vaca y por año. No obstante, estas mejoras pueden quedar relegadas por la aparición de problemas como el bajo desempeño reproductivo de los animales. Tanto en sus formas clínica como subclínica, la mastitis está ligada al estrés de la vaca lechera y tal asociación perjudica considerablemente su desempeño reproductivo. Los productores ganaderos deberán prestar preferencial atención a la tarea de mejorar las condiciones ambientales y de manejo en sus unidades de producción, dado que tales condiciones son en gran parte las responsables del deterioro de la salud de los animales y repercuten en la aparición de trastornos reproductivos, aumentando de manera importante los costos de producción de la industria lechera.
\end{abstract}

Palabras clave: vaca lechera, mastitis, estrés, reproducción.

\begin{abstract}
Córdova-Izquierdo, A.; Córdova-Jiménez, C.A.; Córdova-Jiménez, M.S.; SaltijeralOaxaca, J.A.; Ruiz-Lang, C.G.; Xolalpa-Campos, V.M.; Cortés-Suárez, S.; GuerraLiera, J.E.: Effect of mastitis and stress on cow reproduction. Rev. vet. 19: 2, 161-166, 2008. Because of the undeniable advances in genetic and nutrition, dairy industry can produce great amount of milk per cow per year more efficiently nowdays. However, these improvements may be overshadowed by the emergence of problems regarding low reproductive performance of animals. Both in its clinical and subclinical forms, mastitis is linked to stress in dairy cattle, and such association undermines their reproductive performance. Livestock producers should give preferential attention to the task of improving the environmental conditions and management of their production units, since such conditions are largely responsible of animal health detriment, and affect the incidence of reproductive disorders, increasing significantly production costs of dairy industry.
\end{abstract}

Key words: dairy cow, mastitis, stress, reproduction.

\section{Importancia de la mastitis en la reproducción animal}

La salud de la ubre es determinante para el buen desempeño reproductivo de las hembras, principalmente en las especializadas en la producción de leche. Se sabe que la prevención de las mastitis en lactancias tempranas, mejora la eficiencia reproductiva de los animales en términos de los días al primer servicio postparto, días para la concepción, servicios por concepción y días de intervalo entre partos ${ }^{28}$.

Recibido: 3 mayo 2008 / Aceptado: 4 julio 2008
Las mastitis declaradas antes del servicio, ya sean subclínicas o clínicas, pueden reducir el porcentaje de concepción hasta alrededor del $50 \%$. De esta manera, puede aseverarse que existe una correlación negativa entre la presencia de mastitis y el desempeño reproductivo, principalmente en vacas de alta producción ${ }^{20}$.

Las endotoxinas generadas por los agentes Gram negativos productores de mastitis, pueden favorecer la síntesis de prostaglandinas $\mathrm{F} 2 \alpha$, provocando perturbaciones reproductivas relacionadas con anormalidades del ciclo estral, intervalos entre estros alterados y menor duración de la fase lútea del ciclo estral, cuya repercusión está relacionada con desórdenes en los 
patrones hormonales, menor duración de la vida del cuerpo lúteo (luteólisis), desarrollo folicular, presencia de muertes embrionarias tempranas y compromiso del ambiente uterino ${ }^{6,21,22}$.

En las unidades de producción animal especializadas en la producción de leche, es determinante contar con un buen programa de manejo higiénico-sanitario en todas las áreas, instalaciones confortables y correcto funcionamiento del equipo de ordeño. La mastitis y la reproducción animal, son dos aspectos de gran importancia en el ganado productor de leche. Al respecto, en un estudio retrospectivo se valoraron más de 15000 registros provenientes de 26 unidades de producción de vacas lecheras que tuvieron mastitis, descubriéndose que las vacas que sufrieron tal afección en lactancia temprana, presentaron un más largo intervalo partoprimer servicio ${ }^{13}$. Vacas con mastitis en lactancia temprana presentaron retraso en el primer estro y primera ovulación después del parto, en comparación con las vacas que no la padecieron ${ }^{15}$.

En otro estudio se valoró la fertilidad de unas 9000 vacas lecheras, surgiendo que aquéllas que sufrieron mastitis a los 20-40 días antes del servicio, presentaron $45 \%$ de fertilidad; hallándose un $36 \%$ de fertilidad para aquellos animales que registraron mastitis dentro del período anterior a los 20 días del servicio y un $29 \%$ de fertilidad en los animales que padecieron la mastitis dentro de las primeras tres semanas después del servicio ${ }^{17}$. Los días a primer servicio postparto que repercuten sobre los días abiertos, también se ven alterados con la presencia de mastitis. En una investigación se indicaron datos de primer servicio postparto de 94 días para aquellas vacas que presentaron mastitis dentro de los primeros cinco meses postparto y de 71 días para las que la presentaron después del servicio ${ }^{2}$. También se evidenció que los días abiertos fueron de 114 días para aquellas vacas que presentaron mastitis antes del primer servicio postparto, 137 días para las que sufrieron mastitis entre el primer servicio y la gestación y de 92 días para las vacas sanas.

Existen datos que indican que la reproducción de las vacas lecheras no solo es afectada por la mastitis clínica sino también por la forma subclínica. En una investigación se comunicaron 85 días abiertos para vacas sanas, 108 para aquellas que padecieron mastitis subclínica antes del primer servicio postparto, 91 días cuando la sufrieron después; 110 días cuando tuvieron mastitis clínica antes del servicio y 144 días cuando el padecimiento ocurrió después ${ }^{28}$.

Por otro lado, el cambio de mastitis subclínica a clínica también afecta al desempeño reproductivo: 101 días abiertos cuando esto ocurre antes del servicio y 196 si ocurre después. La presencia de mastitis, ya sea subclínica o clínica en las unidades de producción lechera, también repercute sobre la presencia de abortos, sobre todo durante el primer mes de gestación, tiempo en el cual las vacas pueden ser dos veces más susceptibles a esta falla reproductiva ${ }^{23}$.

\section{El estrés en el deterioro del desempeño reproductivo}

El estrés es el resultado del confinamiento y de una visión ambiciosa y poco humanitaria por parte del hombre que, habitualmente guiado por el interés de mejorar la producción, ha intentado incluso domesticar especies que no pudieron adaptarse sanamente al ambiente impuesto. En la actualidad se ha llegado a la conclusión que el estrés es uno de los factores medioambientales de manejo que más afecta la producción animal, al relacionarse estrechamente con los agentes patógenos que atentan contra la salud de los animales.

El hombre juega un papel muy importante en la generación del estrés, él puede proveer los medios para aliviar, eliminar o modificar cualquier potencial agente estresante ${ }^{5}$. Algunos de los criterios que se han utilizado para indicar la presencia de estrés en los animales son los niveles de hormonas tiroideas, corticosteroides plasmáticos o urinarios, catecolaminas y/o sus metabolitos, ácido láctico, enzimas séricas, valores de glucemia, alteraciones en la frecuencia cardiaca y respiratoria o recuento de células somáticas en leche.

Los mamíferos responden a cambios ambientales mediante mecanismos de adaptación regulados por hormonas como adrenocorticotropina, glucocorticoides y catecolaminas. Bajo condiciones de estrés, existe un efecto negativo de retroalimentación de progesterona sobre la hormona luteinizante, elevándose los niveles de cortisol, prostaglandina F2 $\alpha$ y ACTH. También se registran aumentos de los valores de epinefrina y norepinefrina ${ }^{5}$. Estos cambios afectan negativamente la función reproductiva de los mamíferos ${ }^{8}$. El estrés puede ser calórico, nutricional o por manejo.

Estrés calórico. La radiación solar, la velocidad del viento, la temperatura del aire y la humedad ambiental son factores que tienen que ver con la presentación del estrés calórico. Las primeras respuestas reproductivas al estrés calórico son la disminución de la intensidad del estro y -como consecuencia- la baja fertilidad. Se sabe que las hembras mamíferas son más sensibles al estrés calórico durante los 12 días anteriores al estro, aumentando esta sensibilidad durante los dos días previos al mismo ${ }^{14}$.

En otros estudios se encontró que las altas temperaturas provocan ausencia de libido y afectan también la cantidad y calidad seminal ${ }^{5}$. Además, reducen la presentación de calores y cuando se presentan, son menos intensos y de duración más corta. Si la hembra fue fecundada, la posibilidad de mortalidad embrionaria es alta ${ }^{24}$. Se afirma la existencia de un efecto retrasado del estrés calórico en la esteroidogénesis folicular, así como una disminución en la esteroidogénesis, 20 y 26 días después, en folículos preovulatorios de tamaño medio ${ }^{25}$. Además, las células de la teca se encontraron más susceptibles que las células de la granulosa y éstas expresaron baja producción de estrógenos en los folículos de tamaño medio y baja viabilidad en los folículos 
preovulatorios. En términos de producción de esteroides, las células de la teca parecieron ser consistentemente susceptibles al estrés calórico y expresaron un efecto de transporte sobre la producción de andrógenos en ambos tipos de folículos.

Cuando la temperatura corporal supera $40^{\circ} \mathrm{C}$, los folículos desarrollados pueden sufrir severos daños y convertirse en no viables ${ }^{7}$; a su vez, los aumentos de progesterona inhiben la liberación de LH durante el estro, interrumpiendo el proceso de ovulación ${ }^{16}$. Una investigación reveló que el estrés calórico reduce el tamaño del folículo dominante de la primera y segunda onda folicular ${ }^{25}$. Se postula que exponiendo a las hembras mamíferas domésticas al estrés calórico durante el estro, se altera la dinámica folicular y disminuye el predominio folicular en el estro siguiente. El folículo preovulatorio es un componente clave en el sistema reproductivo y el deterioro de su función durante el estrés calórico puede desencadenar otros efectos, tales como distorsión en la secreción de gonadotropinas y subdesarrollo del cuerpo lúteo y del embrión, cambios que resultan en una baja fertilidad ${ }^{30}$.

Experiencias in vitro sugieren que la exposición de las hembras domésticas preñadas a altas temperaturas ambientales y de humedad, habituales durante los meses de verano, pueden perturbar el balance entre los factores bioquímicos y endometriales responsables del mantenimiento de la preñez. La composición lipídica de las membranas de los ovocitos se deteriora con mayor frecuencia en verano que en invierno ${ }^{25}$. Un aspecto relevante para contrarrestar el efecto del estrés calórico sobre la calidad del ovocito y el desarrollo embrionario, es el posible uso de antioxidantes, para aumentar la resistencia, tanto del ovocito como del embrión. Tiroxina y triyodotironina pueden disminuir los efectos del estrés calórico ${ }^{16}$. Vacas sometidas a bajas temperaturas durante algunos días después de la gestación, mejoraron las tasas de preñez en comparación con hembras que padecieron estrés calórico ${ }^{11}$.

Se ha demostrado que en períodos de severo estrés calórico, solamente un 10-20\% de las inseminaciones pueden resultar en preñeces normales ${ }^{7}$. En Israel, los índices de concepción durante los meses de invierno alcanzan más de $50 \%$, mientras que durante el verano pueden bajar hasta el $20 \%{ }^{31}$. Se afirma que la raza Jersey es más tolerante al estrés calórico que la Holstein. Trabajos realizados en Estados Unidos indican que las vacas Holstein y Jersey establecidas en lugares cálidos, expresan signos de estro tan sólo durante 12 a 13 horas, lo que significa una diferencia de 5 a 6 horas menos que la duración normal del estro de los animales de lugares templados ${ }^{31}$.

Las vacas lecheras con capa negra absorben más calor de los rayos solares, que las vacas de capa blanca 18,19 . Al respecto, se sabe que la temperatura ambiental durante la cual se ve afectada la eficiencia reproductiva de las vacas, va desde los 21,1 a los $32,2^{\circ} \mathrm{C}$; por ello las vacas necesitan ser "enfriadas" tanto como sea posible durante el verano, con el fin de elevar la fertilidad ${ }^{30}$.
En Florida, las condiciones de calor y humedad propias del verano pueden reducir hasta un $10 \%$ la tasa de preñez ${ }^{1}$. Se asegura que las mejores previsiones para controlar los efectos del estrés calórico son la modificación física del ambiente, el mejoramiento genético destinado a lograr razas menos sensibles al estrés calórico y el buen manejo nutricional ${ }^{31}$.

Estrés nutricional. En el ganado bovino, el estrés producido durante el transporte tiene un efecto más perjudicial sobre la fisiología del animal que el estrés causado por la falta de alimentación y bebida, durante un lapso de igual duración. Ello se debe a que el flujo sanguíneo es desviado de los órganos internos hacia los tejidos periféricos, en un intento por reducir la temperatura corporal, mediante el aumento de la pérdida de calor ${ }^{32}$. Este mecanismo ocasiona una disminución del riego sanguíneo destinado a órganos internos como útero, oviductos y ovarios; tal reducción implica escasa disponibilidad de nutrientes para dichos órganos y por lo tanto, deteriora la capacidad funcional ${ }^{19}$.

En hembras estresadas por razones nutricionales el cuerpo lúteo es menos pesado y se constatan cambios en los constituyentes químicos de los fluidos alantoideos, así como degeneración de los embriones de hembras mal alimentadas ${ }^{16}$. Las hembras consumen menos alimento durante las temporadas cálidas; consecuentemente puede existir insuficiencia de nutrientes disponibles después de la producción de leche y por lo tanto, los ovarios no funcionan durante las primeras 6 semanas de lactación ${ }^{29}$.

La vitamina E puede disminuir moderadamente los efectos del estrés calórico durante los meses calurosos, pero no necesariamente aumenta la fertilidad ${ }^{12}$. Un factor previsor podría ser el suministro de alimentos adecuados en cantidad y calidad, los cuales tienen efectos directos sobre la reproducción, así como sobre las alteraciones provocadas por los efectos indirectos del estrés nutricional ${ }^{10}$.

Estrés por manejo. Consiste en interacciones negativas que se establecen entre los animales y sus cuidadores ${ }^{10}$. La forma de maniobrar al ganado puede producirles estrés por manejo inadecuado, el cual puede repercutir en los mecanismos biológicos de la reproducción y de la reacción inmunitaria ${ }^{19}$. Es sabido que las picadas eléctricas, la inmovilización y otros factores de estrés por manejo, debilitan el funcionamiento reproductivo de las hembras.

Existe gran número de situaciones de manejo que pueden provocar estrés afectando seriamente al proceso reproductivo, tales como la movilización excesiva de las hembras antes de ser inseminadas, el uso de perros agresivos en la separación o conducción de los animales, la movilización de los animales en la manga de manejo con diferentes fines y otras ${ }^{29}$. El momento en el que el estrés por manejo produce mayores efectos perjudiciales sobre la reproducción, ocurre poco tiempo después de la presentación del estro ${ }^{19}$. 
Según su temperamento, el bovino puede clasificarse de la manera siguiente: (1) se queda quieto mientras está sujeto, (2) está inquieto, (3) sacude fuertemente la manga de manejo, y (4) sacude de manera violenta la manga de manejo e intenta escapar. En términos generales, los ejemplares de Bos indicus presentan un temperamento más agresivo y nervioso que Bos taurus ${ }^{32}$.

\section{Efecto del estrés sobre el proceso reproductivo}

El bovino es una de las especies más adaptables y posee mecanismos homeocinéticos para mantener las funciones corporales críticas a expensas de cambios en otras funciones fisiológicas, como la reproducción, de tal manera que la función reproductiva es determinada en gran medida por el ambiente ${ }^{31}$. La reproducción es influenciada primordialmente por el estrés ambiental ${ }^{4}$ y el de manejo ${ }^{9}$. El primero incluye a la temperatura exterior, el frío y/o calor, viento y humedad, en tanto que el segundo abarca a la densidad y el flujo de animales, la interacción entre animales de la misma o diferente especie y las condiciones sociales existentes, como estados de angustia psicológica inespecífica, ruido, trauma físico y otras. La combinación de ambos tipos de estrés compromete gravemente la homeostasis ${ }^{5}$. A ellos deberían agregarse algunos tipos de estrés físicos (transporte inadecuado, daño psicológico, aislamiento de los animales) y fisiológicos (hipoglucemia, cambios de la presión sanguínea), todos ellos capaces de generar disfunciones reproductivas en los mamíferos ${ }^{8}$.

El mantenimiento de la temperatura corporal normal se conoce como homeotermia y es de importancia vital para el funcionamiento de los tejidos encefálicos. En el bovino, la variación diurna de la temperatura corporal debe ser de 0,6 a $1,2^{\circ} \mathrm{C}$. Un aumento mayor es signo de enfermedad o de mala adaptación a la elevación térmica. Este rango de temperatura se conoce como zona de confort y varía de acuerdo a la especie y a las razas de animales. Cuando la temperatura corporal es inferior o superior a su grado de confort, el ritmo metabólico disminuye o aumenta, presentándose un desequilibrio en el bienestar animal. El calor corporal es eliminado a través de los mecanismos de radiación, conducción, convección y evaporación ${ }^{31}$. Los efectos del estrés sobre la actividad reproductiva varían según el sexo de los animales.

Efectos en el macho. El estrés ambiental puede provocar baja calidad seminal, la cual está íntimamente relacionada con la reducción de la fertilidad de las hembras, debido probablemente a una combinación de bajas tasas de fertilización y aumento de la mortalidad embrionaria ${ }^{3,5}$.

La exposición directa del testículo a temperaturas altas, provoca alteraciones en ciertas etapas críticas de la espermatogénesis, lo cual también está directamente relacionado con la calidad del eyaculado. Estudios recientes sugirieron que el efecto del estrés térmico sobre la calidad de los espermatozoides puede soslayarse con la puesta en marcha de la tecnología de la congelación seminal; sin embargo, el útero de las hembras puede representar estrés térmico para los espermatozoides ${ }^{26}$.

Efectos en la hembra. El comportamiento sexual y la tasa de fertilidad son los principales indicadores reproductivos que se afectan negativamente en las hembras por el estrés ambiental. Los programas emprendidos con el fin de aumentar la fertilidad de las hembras domésticas, tienen menor éxito en las épocas calurosas que en las templadas ${ }^{3}$. Un aumento de la temperatura uterina de $0,5^{\circ} \mathrm{C}$ durante días calurosos, provoca disminución de la tasa de fecundación. En los bovinos, la exposición de novillas a $32^{\circ} \mathrm{C}$ durante 72 horas después de la inseminación artificial, inhibe el desarrollo embrionario. Sin embargo, se sabe que el $48 \%$ de las hembras mantenidas a $21^{\circ} \mathrm{C}$, puede resultar fertilizada sin problema alguno. Si el estrés térmico se presenta después de los 10 días posteriores al servicio, la fertilidad no es afectada.

La fertilidad en vacas lactantes varía según la estación del año. En invierno disminuye cerca del 50\%; en verano $20 \%$ y en otoño es más baja que en el invierno. Hace algunos años se comunicó que las tasas de concepción en Israel disminuyeron del 52\% en el invierno al $24 \%$ en el verano ${ }^{14}$. En la estación cálida, el $80 \%$ de los estros pueden ser indetectables ${ }^{1}$. Además, se ha indicado que cuando la temperatura rectal de los animales aumenta de 38,5 a $40^{\circ} \mathrm{C}$ a las 72 horas después del servicio o la inseminación, las tasas de preñez pueden disminuir hasta en un $50 \%{ }^{27}$.

Estudios en novillas y vacas han revelado que la disminución de la calidad del ovocito en el período temprano del posparto, está asociada con balance de energía negativo y con bajas condiciones corporales, lo cual se expresa en aumento de embriones subdesarrollados y anormales, teniendo como consecuencia pérdida de embriones en los meses más calurosos del año ${ }^{30}$.

Se afirma que en los bovinos, el desarrollo embrionario es altamente sensible a altas temperaturas, entre los primeros tres a once días después del servicio, adquiriendo más tolerancia a altas temperaturas a medida que el periodo de gestación avanza ${ }^{1,11,12}$. Los embriones obtenidos mediante fecundación in vitro, son más susceptibles al estrés calórico que los obtenidos en condiciones naturales. La mayor pérdida de embriones de bovinos obtenidos de fecundaciones in vitro, ocurre antes de los 42 días, cuando las hembras están bajo estrés calórico ${ }^{1}$.

\section{Recomendaciones para controlar el estrés}

Para evitar el estrés calórico se deberá proporcionar un sistema de ventilación que controle la temperatura corporal, implementar rociadores de agua, proteger a los animales contra las radiaciones solares directas e indirectas por medio de sombras o techos apropiados, proporcionar sombra en comederos y bebederos para aumentar el consumo de alimento en animales con 
estrés calórico, proporcionar a los animales baños por aspersión en las horas más calurosas del día, tratar de tener animales con pelaje blanco ya que disipan mejor el calor que los de capa negra y por lo tanto son menos sensibles al estrés calórico, desarrollar genética adaptada en los animales dado que pueden tornarse menos sensibles al estrés calórico, implantar embriones congelados e inseminar con semen congelado en la época menos calurosa.

Para minimizar el estrés por manejo es conveniente disponer de áreas libres y con sombra en la unidad de producción, proporcionar el área requerida por animal para su mayor confort, bañar a las hembras antes del servicio y a los 3-5 días siguientes, inseminar o dar servicio en períodos menos calurosos, implementar programas de sincronización de estros para programar las inseminaciones o servicios y no aislar a las hembras por mucho tiempo antes de la inseminación artificial o servicio.

Para soslayar el estrés nutricional habrá que balancear adecuadamente las dietas, proporcionando la energía necesaria para compensar eventuales disminuciones de la ingesta, reducir la ingesta de fibra y aumentar la de proteína y energía. En general, se procurará dar bienestar a los animales, evitando el estrés mediante el buen manejo del estado higiénico-sanitario y nutricional. Diagnosticar y prevenir de manera oportuna la presencia de mastitis, a través del uso de una buena rutina de ordeño, cuidando la higiene y el buen estado de las máquinas ordeñadoras, así como usando buenos selladores. El manejo dietario debe contemplar la suplementación con minerales como selenio, calcio y fósforo, además de vitaminas $\mathrm{A}, \mathrm{C}$ y E, cuyo poder antioxidante resulta fundamental para el mantenimiento de la salud de los animales.

\section{CONCLUSIÓN}

La presencia de mastitis tanto subclínica como clínica en las unidades de producción lechera, está ligada al estrés de los animales. Tal conjunción es perjudicial para el desempeño reproductivo de las vacas, ya que aumenta los días de intervalo parto-primer servicio, disminuye la fertilidad, incrementa los días abiertos y servicios por concepción y acrecienta la susceptibilidad al aborto temprano, por lo cual el período entre partos se extiende de manera significativa.

\section{REFERENCIAS}

1. Ambrose JD, Drost M, Monson RL, Rutledge JI, Leibfried-Rutredge ML, Thatcher MJ, Kassa T, Binelli M, Hansen PJ, Chenoweth PJ, Thatcher WW. 1999. Efficacy of timed embryo transfer with fresh and frozen in vitro produced embryos to increase pregnancy rates in heat-stressed dairy cattle. J Dairy Sci 82: 2369-2376.

2. Barker AR, Schrick, FN, Lewis, MJ, Dowlen, HH, Oliver, SP. 1998. Influence of clinical mastitis during early lactation on reproductive performance of Jersey cows. $J$ Dairy Sci 81: 1285-1290.

3. Chemineau P. 1993. Medio ambiente y reproducción animal. Disponible en: www.acontece.com.ar/0113.htm.

4. Córdova IA, Guerrero MJ, Saltijeral OJ, Muñoz MR, Pérez GJ. 2003. Estrés ambiental en la reproducción animal. Prod Anim 191: 49-58.

5. Coubrough RI. 1985. Stress and fertility. A review. J Vet Res 52: 153-156.

6. Cullor JS. 1990. Mastitis and its influence upon reproductive performance in dairy cattle. Proceedings Int Symp Bov Mastitis, Indianapolis, p. 176-180.

7. Dave L. 1999. Manejo del estrés de calor. Disponible en: http://www.accelgen.com/spanish/nov_heat150.html.

8. Dobson H, Smith RF. 1995. Stress and reproduction in farm animals. J Reprod Fertil Suppl 49: 451-461.

9. Dobson H, Smith RF. 2000. What is stress, and how does it affect reproduction? Anim Reprod Sci 2: 743-752.

10. Dobson H, Tebble JE, Smith RF, Ward WR. 2001. Is stress really all that important?. Theriogenology 55: 6573.

11. Ealy AD, Drost M, Hansen PJ. 1993. Developmental changes in embryonic resistance to adverse effects of maternal heat stress in cows. J Dairy Sci 76: 2899-2905.

12. Ealy AD, Arechiga CF, Bray DR, Risco CA, Hansen PJ. 1994. Effectiveness of short-term cooling and vitamin $E$ for alleviation of infertility induced by heat stress in dairy cows. J Dairy Sci 77: 3601-3607.

13. Eicker SW, Grohn YT, Hertl JA. 1996. The association between cumulative milk yield, days open, and days to first breeding in New York Holstein cows. J Dairy Sci 79: 235-241.

14. Gilad E, Meidan R, Berman A, Graber Y, Wolfenson D. 1993. Effect of heat stress on tonic and GnRH-induced gonadotrophin secretion in relation to concentration of oestradiol in plasma of cyclic cows. J Reprod Fertil 99: 315-321.

15. Huszenicza G, Jánosi S, Kulcsár M, Kóródi P, Dieleman SJ, Bartyik J, Rudas P, Ribiczei-Szabó P. 1998. Gramnegative mastitis in early lactation may interfere with ovarian and certain endocrine functions and metabolism in dairy cows. Reprod Dom Anim 33: 147-153.

16. Lee CN. 1993. Environmental stress effects on bovine reproduction. Vet Clin North Am Food Anim Pract 9: 263273.

17. Loeffler SH, de Vries MJ, Schukken YH. 1999. The effects of time of disease occurrence, milk yield, and body condition on fertility of dairy cows. J Dairy Sci 82: 2589 2604.

18. Madison, WI. Ullah G, Fuquay JW, Keawkhong T, Clark BL, Pogue DE, Murphey EJ. 1996. Effect of gonadotropin-releasing hormone at estrus on subsequent luteal function and fertility in lactating Holsteins during heat stress. J Dairy Sci 79: 1950-1953.

19. Mazzucchelli F, Tesouro MA. 2000. Influencia del estrés sobre la eficiencia reproductora del ganado vacuno de leche. Disponible en: www.redvya.com/veterinarios/ veterinarios/especialidades/ bovino/especialista/Articulo03. htm. 
20. Moore DA, Cullor JS, BonDurant RH, Sischo WM. 1991. Preliminary field evidence for the association of clinical mastitis with altered interestrus intervals in dairy cattle. Theriogenology 36: 257-265.

21. Moore DA, O'Connor ML. 1993. Coliform mastitis: its possible effects on reproduction in dairy cattle. Proceedings Natl Mastitis Counc, Kansas City, p. 162-166.

22. Oliver SP, Schrick FN, Hockett ME, Dowlen HH. 2000. Clinical and subclinical mastitis during early lactation impairs reproductive performance of dairy cows. Proceedings Natl Mastitis Counc, Cleveland, p. 34-51.

23. Risco CA, Donovan GA, Hernandez J. 1999. Clinical mastitis associated with abortion in dairy cows. J Dairy Sci 82: 1684-1689.

24. Rodríguez JB. 1997. Como minimizar el estrés calórico del verano en vacas lecheras. On Line: http://patrocipes. uson.mx/patrocipes/invpec/ranchos/RA0085.html.

25. Roth Z, Arav A, Bor A, Zeron Y, Braw-Tal R, Wolfenson D. 2001. Improvement of quality of oocytes collected in the autumn by enhanced removal of impaired follicles from previously heat-stressed cows. Reproduction 122: $737-744$
26. Rutledge JJ. 2001. Use of embryo transfer and IVF to bypass effects of heat stress. Theriogenology 55: 105-111.

27. Ryan DP, Blakewood EG, Iynn JW, Munyakazi I, Godke RA. 1992 . Effect of heat-stress on bovine embryo development in vitro. J Anim Sci 70: 3490-3497.

28. Schrick FN, Saxton AM, Lewis MJ, Dowlen HH, Oliver SP. 1999. Effects of clinical and subclinical mastitis during early lactation on reproductive performance of Jersey cows. Proceedings Natl Mastitis Counc, Arlington, p. 189-190.

29. Varner MA. 1998. Estrés y reproducción. Disponible en: http://www.unt.edu.ar/ faz/labrydea/Estres.htm.

30. Wolfenson D, Lew BJ, Thatcher WW, Graber Y, Meidan R. 1997. Seasonal and acute heat stress effects on steroid production by dominant follicles in cows. Anim Reprod Sci 47: 9-19.

31. Yabuta O, Kunio A. 2000. El estrés calórico en el ganado lechero. Disponible en: http://fmvzl.uat.mx/Investigacion/ memorias/principal7.htm.

32. Zapiola M. 1998. La reducción del estrés del manejo mejora la productividad y el bienestar animal. http://www. grandin.com/spanish/reduccion.estres.manejo.html. 